5. Frequency of Dose.

If 3 or 4 grains be adopted as a dose, and 16 grains as needful daily, it means that four or five doses a day must be given. For a full-sized adult this implies an amount just bordering on one sufficient to cause nervous symptoms, varying with the patient-symptoms which I always try to avoid. This will effect a fairly continuous, yet mild, quininization of the patient's system, with the idea of steadily lowering the vitality of the plasmodia till they become unable to reproduce their kind-just the opposite of the therapia sterilisans magna, or knock-out blow. It is this great evenness and persistence of treatment that I venture to think affords this, and one which might be labelled "therapia sterilisans perstans." The doses should be given as far as possible at equal intervals, and therefore one should be given during the night, if possible, or at least the last thing at night, and another the first thing in the morning.

\section{Coincident Treatment.}

This consists in most cases of preliminary treatment with an aperient, mostly calomel. In view of the action of the latter in causing "bilious" motions, whether due to lessening catarrh in the region of the duodenum, etc., by ácting as an antiseptic, or otherwise, the possibility of its aiding indirectly the absorption of quinine is evident in view of MacGilchrist's experiments. He found that the alkaloid quinine in the crystalline form is soluble 1 in 625 of blood serum (that is, three times as soluble as in water), but is soluble 1 in 183 of bile (that is, about nine times as soluble as in water). Against this it is often assumed that the alkaline secretions of the liver, pancreas, and intestines precipitate the quinine, though, as MacGilchrist showed, quinine glycocholate is soluble in excess of bile. There may be in addition to the antiseptic and characteristie aperient action of calomel another due to its increasing the mucous secretions in the bile ducts of the liver (as occurs with the salivary and intestinal secretions), and so removing the blocking by the malaria-thickened bile. In some cases of persistent headaches in patients affected with malaria calomel acts very quickly and thus I have not needed the help of acetyl-salicylic acid, gelsemium, etc., which at best act symptomatically.

I may now refer to some prescriptions in which small doses of quinine are alleged to be effective.

1. Warburg's Tincture is a very complicated mixture containing some fifteen ingredients. It contains in each half-ounce 4 grain of quinine sulphate, and as only two doses were to be given, in all only 8 grains were administered. But with each dose there are given 6 grains of aloes - that is; within half an hour, 12 in allrather severe treatment. In view of the readiness with which a chill seems to bring on an attack of malarial fever the diaphoretics contained in the tincture are of possible value; they may be expected also to act beneficially as gastro-intestinal antiseptics. The alcohol is evidently a powerful solvent medium, and beyond that its action need not specially concern us. This preparation has been abanneed not specially concern us. This preparation has been aban-
doned since the aperient action, at least in some cases, must be rather strong; hence the aloes, if desired, is omitted in the various preparations supplied commercially, thus, perhaps, removing the soul of the efficiency ascribed to it. The complicated form makes it expensive, many of the ingredients are practically unobtainable and their effects are unknown; it is said to depress the heart and has an appalling taste.

2. H uxham's Tincture was the forerunner of the compound tincture of cinchona, the composition of which has varied since its first introduction. The dose was 1 to 4 fluid drachms. Originally it was made with pale, but is now made with red, cinchona bark the other constituents being orange peel and serpentary and saffron (now, omitted). The serpentary in small doses acts as an aromatio bitter, diaphoretic and diuretic; in larger doses as an emetic and iperic apert cent. cinchona bark, cinchona alkaloid. At four doses a day this amounts to 6 grains of cinchona alkaloids with some possible help from antiseptics and the compound tincture of cinchona is only half to one fluid drachm.

3. E. T. Simpson (Civil Surgeon of Oudh, India) recommends in all acute cases where diarrhoea is not present:

$$
\begin{aligned}
& \text { Magnesiae sulphas } \\
& \text { Liq. ammoniae aceta } \\
& \begin{array}{l}
\text { Quininae sulphas } \\
\text { Aquam camphorae }
\end{array} \\
& \text { Sig. : One ounce every four hours. } \\
& \begin{array}{ll}
\ldots & \text { dr. if } \\
\ldots . & \text { f. } .0 z . j \\
\ldots & \text { gr. } x \mathbf{r v}
\end{array} \\
& \text {....ad fr. Xz oz vilj }
\end{aligned}
$$

He claims that 90 per cent. of cases recover under this treatment. He employs a somewhat similar prescription for chronic tertian. 4. Finally, there is Forbes Leslie's recommendation of calomel only:

Method Recommended.

The foregoing will make clear the principle-namely, the more or less continuous coincident administration of an aperient, preferably calomel-upon which I base the method which I have for many years adopted and which is as follows :

1. Give a small dose of calomel at once, 1 grain, perhaps repeated in an hour or so; this single dose is to be administered each night at bedtime. I prefer the pill form as less liable than that of a powder to cause mercurialism in the mouth. This should be followed early next morning by a saline aperient. This will help release of bile into the duodenum and by its antiseptic and aperient action without irritation cleanse the bowels. The continual administration of calomel, in doses insufficient to risk distinct mercurialization, is to my mind extremely, indeed I think essentially, important; though in certain circumstances a stronger dosage for the purpose of thinning the thickened bile might be desirable. The rapidity, however, with which the calomel aids quinine indicates a local gastro-intestinal action rather than one due to absorption.

2. Give the quinine in 3 or 4 grain doses according to the size and strength of the (adult) patient, as a pill; the small dose and form avoid irritation of the stomach.

3. Give the hydrochloride; this is in order to secure rapid absorption with minimum of gastric irritation.

4. Give the doses an hour or more before meals, and at bedime; this is in order to secure rapid absorption.

5. Give with each dose 3 or 4 ounces of water; the amount of fluid, by diluting the quinine freely, prevents disturbance of the stomach and encourages rapid removal from it.

6. Continue the treatment for two months.

I have never failed in this way rapidly to relieve urgent symptoms; generally there is no attack or at most only a slight one in the first week of treatment, none afterwards in my experience. Those few cases which I have followed a year or two after the treatment have been free from attacks and have felt unusually well as compared with their general condition between the attacks before treatment. But, no more with this, perhaps, than with other treatments can one promise an absolute cure. I claim, then-

1. That the method is in no sense unpleasant, except as regards taking the salines, though even these might be replaced by other aperients.

2. That it is easily carried out, being very simple. The patient can go on with his work, not needing to go into hospital

3. That the drugs are easily obtained, are portable, and keep well. 4. That if the result is not perfect it at least is produced more rapidly than by the ordinary routine, and in general is much more rapidily tha nerves due to quinine, for, indeed, the slightest symptoms of such disorders are taken as the indication for lessening the dose.

Although my experience is limited to chronic cases it is quite possible that the principles of this treatmentiare adaptable at least in some measure to acute cases.

REFERENCES.

1 Journ. of the R.A.M.C., February, 1922. ${ }^{2}$ Manual of Tropical Medicine, 1919. "Quinine and its Salts, their Solubility

\section{THE AUTOGENOUS PEG GRAFT IN CERTAIN FRACTURES OF THE FEMUR.}

\section{BY \\ WALTER MERCER, F.R.C.S.EDIN., DDIYBURGH.}

From a study of the end-results of simple fractures of the shaft of the femur it would appear that in spite of a great variety of methods of treatment there is still scope for one which will ensure a more perfect end-result.

The disabilities resulting from fracture of the shaft are from two main causes-bad alignment and knee-joint trouble.

\section{Faulty Alignment.}

The shortening is maintained by the pull of the muscles irritated by the bony ends and by the distension of the fascial planes with haemorrhage and exudate. This is usually considerable at first, and attempts at manual reduction frequently fail. Oblique fractures make the retention of the position very difficult. The usual procedure is to 
attempt manipulation if there is marked shortening, and if retention is difficult extension is applied to the femur by one of the various methods.

The extraordinarily careful adjustment of the line of pull and the delicate nature of the means of retention of the fragments mean constant watching and attention; in civil hospitals the surgeon has not the constant stream of such cases that were seen in military hospitals, and minor defects result. Manipulation followed by traction with a considerable weight frequently fails to overcome the muscles, so that shortening and overriding persists, and when reduction is successful and retention is attempted by extension the presence of the necessary apparatus makes it difficult to carry on the movements of the joints and the massage which are so useful to the injured limb.

\section{Limitation of Knee Movements.}

The commonest cause of the limitation of movement of the knee is the long-continued immobilization, as was so evident in many of our soldiers returned from German prisoner-of-war hospitals, where they had not been looked after. It is said that immobilization will not limit the movements of a healthy joint. This may be so, but it allows tendons and muscles to undergo fibrosis and shortening, and the end-result is the same-a limitation in the extent of joint movement. This fibrosis is due in many cases to the actual wounding of the quadriceps by the bony fragments. When healing takes place fibrous tissue forms; this limits the relaxation of the muscle and also tacks it down to the fracture. Actual adhesions in the joint are rarely considerable enough to prevent their being gradually broken down, nor is any capsular shortening ever very troublesome.

We should aim at some method by which complete reduction of the deformity is obtained and retained with some degree of certainty and yet without any cumbersome appliance that will prevent active movements and massage to the affected parts. An autogenous intramedullary peg bone graft as here described fulfils these conditions. This graft of live bone ensures alignment in a good position and maintains fixation while the osteogenetic functions produce a greater amount of internal callus, and the patient is allowed a greater freedom of movement at an earlier date. The peg stimulates bone production, and this new bone fills up between the peg and the parent bone till a solid mass is produced. This increases in strength according to the demand put upon it, and it is of great importance to get movements started early so that the new tissue may be "taught" its function. It is not contended that the peg is a permanence, as with complete bony union the shaft generally resumes its normal shape and a complete medullary cavity is reproduced. This is even produced when a long gap of 4 to 5 inches in the forearm bones is filled with the anterior surface of the tibia. It takes some years to produce, but it is the eventual result.

The essential feature of every case is that there is no wound of the skin that might lead to sepsis in the operative field, hence it is practically only in a simple fracture that the method can be used.

In many oblique fractures where both reduction and retention are difficult and where external immobilization is necessary afterwards to retain the position an autogenous peg graft would make the after-treatment easier and the end-result better. It would also be the operation of choice in that class of fracture in which reduction cannot be secured otherwise than by open operation. The obstacle to reduction would appear to be either the pull of strong muscles irritated by the fragments or oedema expanding and so shortening the fascia.

\section{Mode of Using a Peg Graft.}

If operative treatment is deemed essential it is necessary to wait only until any shock has disappeared. It is better to give the operative field a very complete preparation; this is risually begun two days before the operation, and after the usual shaving, washing with spirit, soap, and lysol, spirit is applied and then $1 / 2$ per cent. picric acid; this is found to be less irritating to the skin than iodine and equally effective. The skin of the same leg over the crest of the tibia is prepared equally carefully.

The fracture is exposed by an incision 5 to 7 inches long over its lateral aspect. It is a matter of choice whether sterile towels should be clipped round the wound, but where there is any doubt about the skin the towels should certainly be used. The blood clot, including that in the marrow cavity, is cleared away as freely as possible. Traction is applied to the limb by a second assistant, and the deformity is reduced by the levering aid of a strong file and Lane's T-shaped lever with serrated edges. It is essential to obtain this preliminary reduction so as to stretch the muscles and make the reduction easier when putting in the graft. The leg is now adducted at the site of fracture and the ends protruded from the wound so that the medullary cavity may be prepared. The size of this is increased in its transverse axis by the reamer attached to the Albee saw. The actual calibre is not increased in all directions as the peg graft to be introduced is flattened rather than round. This reaming is done to a greater length in one of the fragments, that length being the same as the graft to be cut. The length in the other fragment need only be half the length of the graft.

The peg graft is cut from the anterior surface of the tibia and should be about 4 to 5 inches long, unless the fracture is oblique, when a greater length is necessary. The breadth should be just less than the already measured breadth of the marrow cavity it has to fit. The periosteum is now stripped from the graft, as this fibrous tissue element prevents union of its underlying bone. A shallow saw cut is made at the mid-point of the graft so that its correct position may be ascertained when fitted.

The graft is now inserted into the upper fragment to practically its full extent; it should not be gripped too tightly by the femur, but should be so loose as to be easily extracted by a pair of Kocher's artery forceps which are clipped on to the terminal $1 / 8$ inch of the graft, which is allowed to protrude. The lower fragment is now got into position by traction on the foot and by leverage, but the thickness of a strong file-about $3 / 8$ inch-is interposed between the bony ends, so that a gap of that amount is left between the bone fragments through which the Kocher's artery forceps are used to " tease " down the graft into position. This is recognized to be correct when the saw cut in the middle of the graft is seen, and then the file is carefully removed while traction is applied. The traction being removed, accurate reduction is obtained and retained by the peg graft. The muscles and fascia are stitched with interrupted catgut and the skin with interrupted silkworm gut. No drainage is necessary, but the stitches are not put in too closely so that the effused blood can get away easily.

Although I used a complete plaster case in some of my earlier cases I do not now consider this necessary, as with ordinary care of a good nursing staff there is little to fear. The leg is accordingly bandaged firmly on a double inclined plane and the plane fixed to the foot of the bed. About the end of the second week the bandage is removed from the leg and the knee extended and some gentle massage given. This is continued once daily right on and the movements gradually increased. By the end of six weeks the splint may be removed and active movements increased by this freedom. Consolidation of the callus is frequently quickened by ionization with calcium salts, while the faradic current to the quadriceps is extremely useful in developing a muscle slightly wasted by disuse. The patient should be taught frequently to contract his quadriceps even though not getting great movement with this contraction, to preclude any possibility of it adhering to callus. He is up on crutches at the end of two months and can get home. In three months a walking calliper is applied and used for other three months, by which time the patient should be saying good-bye to the surgeon. The method is so simple and reasonable that it seems necessary to add the history of only one typical case:

K. B., aged 23, as a result of a motor cycling accident, was K. B., aged 23, as a May 23rd, 1921, with a simple fracture of the lower third of the right femur presenting the usual signs, but 
no crepitus could be made out. There was over 3 inches of shortening. $\boldsymbol{X}$-ray examination showed a transverse fracture at the junction of the lower and middle thirds with about 3 inches the junction of the lower and middle thirds with about 3 inches plane, and on May 24th, under a general anaesthetic, was pulled plane, and on May 24th, under a general anaesthetic, was pulled roller towel round the perineum. Pearson's ice-tong calliper was applied and the patient put back to bed; $x$-ray examination showed that there was still 2 inches of shortening.

On June 5th a further attempt was made under chloroform to correct the displacement of the lower fragments and the leg was put on an inclined plane. This put on an inclined plane. Pearson calliper with a roller towel round the perineum to the head of the bed. $X$ rays still showed the fracture to be in an unsatisfactory position, and the shortening not reduced after fortyeight hours of this weight. It was accordingly decided to operate on the fracture.

\section{Operation.}

On June 11th an incision was made along the lateral aspect of the leg, exposing the site of fracture. There was a considerable amount of blood clot and bruising of muscle at this level. By means of traction on the leg and leverage with a heavy file between means of traction on the leg and leverage with a heavy file between
the ends it was seen to be possible to get the fragments in good the ends it was seen to be possible to get the fragments in good
position. Retention, however, appeared difficult, and I decided to introduce an intramedullary peg bone graft. An incision was made over the tibia on the same side, and a piece of the anterio surface of the tibia about 6 inches long by $3 / 4$ inch broad was removed by Albee's electric saw. The graft was slightly sharpened medulla of both femoral fragments. The graft was then inserted into the two fragments and their position was found to be easily retained by this means. The wounds were then closed with catgut and silkworm gut without drainage; the leg was applied to a Miles's double inclined plane.

Recovery from the operation was uninterrupted and the stitches were taken out on the thirteenth day; knee movements were started at the end of the first week. The inclined plane was left off on the twenty-first day, he was up on the twenty-eighth day, and out on the fifty-second day. No shortening resulted.

The radiogram of the femur taken about two months after the operation showed perfect alignment with complete osseous union and a considerable amount of callus around the fracture. The knee- and ankle-joint movements rapidly improved, the full extension of the knee being the last movement to come, but all were perfect on his discharge, and he now walks without a limp and is able to run and play strenuous games without any difficulty.

\section{Conclusions.}

1. The method by autoplastic peg graft is superior to all other operative measures in that it retains the alignment of the fracture and helps to form new bone, so ensuring union at an earlier date and allowing early active and passive movement.

2. Though requiring a certain skill with the electric saw it is by no means a difficult operation.

3. The peg is eventually absorbed from the marrow cavity, leaving the shaft in the normal condition.

4. It obviates any limitation of knee movements as the joint is moved from the tenth to the twelfth day.

5. The results achieved warrant its much wider application.

\section{LARYNGEAL INTUBATION IN ANAESTHETICS.} BY

STANLEY ROWBOTHAM,

ANAESTHETIST, THE QUEEN'S HOSPITAL FOR FACIAL AND JAW INJURIES, SIDCUP; HONORARY ANAESTHETIST, THE CANCER HOSPITAL AND THE SEAMEN'S HOSPITAL, GREENWICH.

THF method of laryngeal intubation to be described is offered (1) as an alternative, and (2) in some cases as a very necessary adjunct, to intratracheal insufflation where this method of anaesthesia is indicated.

Intubation for the administration of the anaesthetic, when the operation is in the region of the mouth or jaw, is usually performed by means of a Kühn's tube. The indirect method of intubation, however, is not generally so easy nor so certain as that of passing a tube through the glottis by direct vision; moreover, the tube supplied by instrument makers in this country seems to offer a very considerable resistance to free respiration.

The technique of intubation to be described is no more difficult and takes no longer than that for passing a tracheal catheter. The following advantages may be claimed for it:

(a) It completely shuts off the air passages so that no blood or mucus can enter.

(b) It enables gas and oxygen almost entirely to be administered, and, as most operations in this region do not require very great relaxation, the patient can be kept in a lighter zone of anaesthesia without fear of respiratory obstruction, the glottic sphincter being entirely out of action.

(c) $\hat{A}$ soft rubber tube is used which does not injure the cords.

In combination with a tracheal catheter-

(d) To obtain expiration entirely free from all obstruction some form of return airway is usually necessary with intratracheal insufflation. This especially applies to cases where the surgeon is working on the lower jaw.

(e) It prevents bubbling of blood from hindering the surgeon when the operation is in the mouth or nose. In the latter case a post-nasal sponge is unnecessary.

The tube used is of rubber, rather a cheap quality being

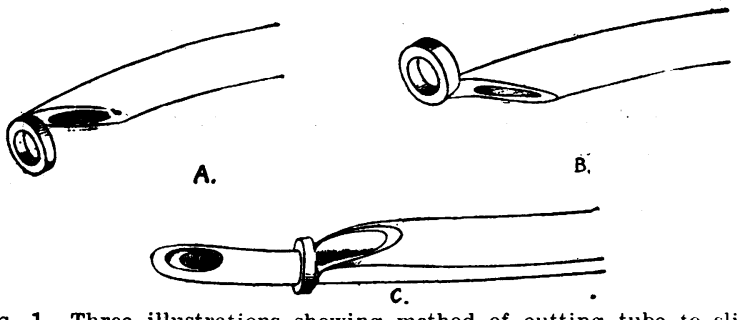

FIG. 1.-Three illustrations showing method of cutting tube to slide along catheter.

preferable because it is not so soft and compressible. The walls are not more than $2 \mathrm{~mm}$. thick.. The size varies with the patient and also with its mode of use, a larger size, of course, being necessary if the patient is to breathe backwards and forwards through it, than if it is merely to be used as a return airway, the entering stream of air being supplied by way of a tracheal catheter. Tubes approximating numbers 22 to 32 French catheter sizes are most generally useful, and the length should be about 8 inches, so as to carry the end well into the trachea. When the tube is to be passed through the nose, its end is cut away in a sloping manner to facilitate its passage through the nose, and a small hole is punche' balf an inch from the end

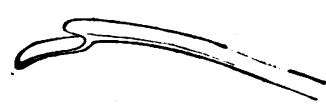

A

Fu. 2.-Two illustrations showing directing rod and method of hooking up tube. to receive the hooked end of the directing rod. When used as a return airway with a catheter passed through the mouth, a tube is cut as in Fig. 1, and the end turned round to form a loop; this slides down the catheter after it has been passed into the trachea, and so is guided through the cords.

The directing rod is a straight metal rod curved slightly upwards and forked at the distal end. The inner prong of the fork is turned upwards and a little outwards so as easily to enter the hole in the tube when catching it up in the pharynx. The outer prong is cut short (Fig. 2).

The direct laryngoscope is used for intubation. When intubating by way of the mouth, the directing rod is hooked into the hole in the distal end of the tube, which is cut as for passage through the nose. The two are held together and passed by the side of the speculum. The upward bend on the directing rod enables the tube to be more easily directed between the cords. As soon as the thin end nof the tube has passed into the glottis it is pushed on alone, and the directing rod gently withdrawn. 